\title{
Analysis of emissions from prebunched electron beams
}

\author{
Jia Qika* \\ National Synchrotron Radiation Laboratory, \\ University of Science and Technology of China Hefei, Anhui 230029, China
}

(Received 18 April 2017; published 24 July 2017)

\begin{abstract}
The emissions of the prebunched electron beam, including the coherent spontaneous emission and the self-amplified stimulated emission, are analyzed by using one-dimensional FEL theory. Neglecting the interaction of the electrons and the radiation field, the formula of the coherent spontaneous emission is given, the power of which is proportional to the square of the initial bunching factor and of the undulator length. For the general emission case of the prebunched electron beam, the evolution equation of the optical field is deducted. Then the analytical expression of the emission power is obtained for the resonant case; it is applicable to the regions from the low gain to the high gain. It is found that when the undulator length is shorter than four gain lengths, the emission is just the coherent spontaneous emission, and conversely, it is the self-amplified stimulated emission growing exponentially. For the nonresonant prebunched electron beam, the variations of the emission intensity with the detuning parameter for different interaction length are presented. The radiation field characters of the prebunched electron beam are discussed and compared with that of the seeded FEL amplifier.
\end{abstract}

DOI: 10.1103/PhysRevAccelBeams.20.070702

\section{INTRODUCTION}

An important basic concept of the free electron laser (FEL) is the bunching. When the electrons pass through the magnetic field of an undulator, they interact with an optical field whose wavelength resonates with the radiation wavelength. Depending on the phase relative to the optical field the electrons experience either a deceleration or an acceleration, thus it leads to an energy modulation of the electrons. As the energy modulated electrons go on traveling in the undulator, the fast and the slow electrons follow the paths of different lengths in the undulator magnetic field. Hence the energy modulation is transformed into a density modulation, i.e. the microbunching on the scale of the optical wavelength is formed. Consequently, the radiation from the microbunched electrons is coherently enhanced.

If prior to injection into the undulator the electron beam is prebunched on the scale length comparable with (at least) or shorter than the resonant wavelength, then the coherent radiation will be developed quickly. The prebunching mode [1-4] can be used for compact FELs, such as intense narrow-band $\mathrm{THz}$ superradiance sources [5-7], as well as high gain x-ray FELs [8-9], to not only shorten the saturation length but also improve the longitudinal coherence.

\footnotetext{
*jiaqk@ustc.edu.cn
}

Published by the American Physical Society under the terms of the Creative Commons Attribution 4.0 International license. Further distribution of this work must maintain attribution to the author(s) and the published article's title, journal citation, and DOI.
In this paper, the emissions of the prebunched electron beam are analyzed by using the one-dimensional FEL theory. We analyze the coherent spontaneous emission and the self-amplified stimulated emission of the prebunched electron beam. We consider the case of a FEL operating with the sustained electron pulse train. The paper is organized as follows: First, the basic equations of the radiation optical field with the prebunched electron beams are presented (Sec. II), and then we deduce the evolution equations of the radiation optical field for the spontaneous emission and the self-amplified stimulated emission of the prebunched electron beam (Sec. III). In Sec. IV we analyze and discuss the behaviors of the FELs with the prebunched electron beam, and compare them with the seeding modes. Finally, Sec. V is devoted to concluding remarks.

\section{BASIC EQUATION}

Under the slowly varying envelope approximation the optical field equation and the ponder motive phase equation in one-dimensional FEL mode are [10-15]

$$
\begin{aligned}
& \frac{d \tilde{a}_{s}}{d z}=\frac{\lambda_{s} r_{e} a_{u} f_{c} n_{e}}{\gamma}\left\langle e^{-i \phi}\right\rangle, \\
& \frac{d^{2} \phi}{d z^{2}}=-\frac{2 k_{u} k_{s} a_{u} f_{c}}{\gamma^{2}} \operatorname{Re}\left(\tilde{a}_{s} e^{i \phi}\right),
\end{aligned}
$$

where $\tilde{a}_{s}=a_{s} e^{-i \varphi_{s}}, \quad a_{s}=e E_{s} /\left(m c^{2} k_{s}\right) \quad$ and $a_{u}=$ $e B_{u} /\left(m c^{2} k_{u}\right)$ are dimensionless vector potential of the $r m s$ radiation field $E_{s}$ and undulator field $B_{u}$, respectively; $k_{s}=2 \pi / \lambda_{s}, k_{u}=2 \pi / \lambda_{u}$ are the corresponding wave numbers; $\varphi_{s}$ is the phase of the radiation field; $r_{e}$ is the classical electron radius; $n_{e}$ and $\gamma$ is the density and the energy 
of the electrons; $f_{c}$ is the undulator coupling factor: for the circularly polarized helical undulator $f_{c}=1$; for the linearly polarized planar undulator it is a difference of the two Bessel functions $f_{c}=J_{0}(\zeta)-J_{1}(\zeta), \quad \zeta=$ $a_{u}^{2} / 2\left(1+a_{u}^{2}\right)$; the angular bracket represents the average over the electron's initial phases and initial phase velocities. The ponderomotive phase of electron is $\phi=\left(k_{s}+k_{u}\right) z-$ $\omega_{s} t$, it can be written as

$$
\phi=\phi_{0}+\phi_{0}^{\prime} z+\Delta \phi,
$$

where $\phi_{0}$ and $\phi_{0}^{\prime}$ are the initial phase and the phase velocity (i.e. the detuning parameter, it is proportional to the resonance energy offset of the electron), $\Delta \phi$ is the phase change due to interaction with the optical field and can be given from Eq. (2):

$$
\begin{aligned}
\Delta \phi & =-2 k_{s} k_{u} a_{u} f_{c} \operatorname{Re} \iint \frac{\tilde{a}_{s} e^{i \phi}}{\gamma^{2}} d z^{\prime \prime} d z^{\prime} \\
& =-2 k_{s} k_{u} a_{u} f_{c} \operatorname{Re} \int_{0}^{z}\left(l-z^{\prime}\right) \frac{\tilde{a}_{s} e^{i \phi}}{\gamma^{2}} d z^{\prime} .
\end{aligned}
$$

When the optical field is not strong, the electron phase change due to interaction with the optical field is not large, then the exponential term in Eq. (1) can be linearized, thus we have

$$
e^{-i \phi}=e^{-i\left[\phi_{0}+\phi_{0}^{\prime} z+\Delta \phi\right]} \cong e^{-i\left[\phi_{0}+\phi_{0}^{\prime} z\right]}(1-i \Delta \phi) .
$$

Here in the expression of the $\Delta \phi$, we neglect the perturbation part of the phase in the exponential of the integral. Inserting Eqs. (4) and (5) into Eq. (1) and averaging over the initial distribution of electrons in the phase space, we obtain

$$
\begin{aligned}
\frac{d \tilde{a}_{s}}{d z}= & I_{T}\left\langle e^{-i\left(\phi_{0}+\phi_{0}^{\prime} z\right)}\right\rangle \\
& -\left(2 k_{u} \rho\right)^{3}\left[\left\langle\frac{\partial}{\partial \phi_{0}^{\prime}} \int_{0}^{z} e^{-i \phi_{0}^{\prime}\left(z-z^{\prime}\right)} \tilde{a}_{s} d z\right\rangle\right. \\
& \left.-i\left\langle\int_{0}^{z}\left(z-z^{\prime}\right) e^{-i\left[2 \phi_{0}+\phi_{0}^{\prime}\left(z+z^{\prime}\right)\right]} \tilde{a}_{s}^{*} d z\right\rangle\right] .
\end{aligned}
$$

Here $I_{T}=r_{e} \lambda_{s} a_{u} f_{c} n_{e} / \gamma$, it is proportional to the transverse current intensity, $\rho$ is the Pierce parameter, $(2 \gamma \rho)^{3}=$ $r_{e} n_{e} \lambda_{u}^{2} a_{u}^{2} f_{c}^{2} / 2 \pi$. For the usual FEL's theoretical analysis, an electron beam with uniform initial phase distribution is assumed, i.e. no initial bunching, therefore the first term (and the third term) on the right-hand side of the above equation is neglected and only the second term is considered. But for the electron beam being initially prebunched, the first term cannot be neglected on the right-hand side of Eq. (6).

Considering the monoenergy electron beam and neglecting the second-harmonic initial bunching, Eq. (6) becomes $\frac{d \tilde{a}_{s}}{d z}=I_{T} \tilde{b}_{0} e^{-i \phi_{0}^{\prime} z}-\left(2 k_{u} \rho\right)^{3} \frac{\partial}{\partial \phi_{0}^{\prime}} \int_{0}^{z} e^{-i \phi_{0}^{\prime}\left(z-z^{\prime}\right)} \tilde{a}_{s} d z$

where $\tilde{b}_{0}=\left\langle e^{-i \phi_{0}}\right\rangle$ is the initial bunching factor.

\section{RADIATION FROM PREBUNCHED ELECTRON BEAM}

First, we consider the spontaneous emission of the prebunched electron beam, the case in which the interaction between the electrons and the radiation field can be neglected, and without seeding $\left(a_{s 0}=0\right)$. Therefore we neglect the changes of the electron phase due to the interaction with the optical field and only consider the first term on the right-hand side of Eq. (7). We get the radiation field and the power:

$$
\begin{gathered}
\tilde{a}_{s}=I_{T} \tilde{b}_{0} L \sin c \frac{\phi_{0}^{\prime} L}{2} e^{-i \frac{\phi_{0}^{\prime}}{2} L}, \\
P=\left(2 k_{u} \rho L\right)^{2} b_{0}^{2} \rho P_{e} \sin c^{2} \frac{\phi_{0}^{\prime} L}{2},
\end{gathered}
$$

where $P_{e}=I \varepsilon_{e} / e$ is the power of the electron beam, $I$ and $\varepsilon_{e}=m c^{2} \gamma$ are the peak current and the energy of the electron beam, respectively.

For the ideal case that the electrons are full modulated, namely all the electrons are in the same phase, the bunching factor $b_{0}=1$, and the emission is completely coherent (superradiance), then from Eq. (8) it has (consider the resonant case $\phi_{0}^{\prime}=0$ )

$$
P_{\text {sup }}=\left(2 k_{u} \rho L\right)^{2} \rho P_{e} .
$$

Thus the superradiance power is proportional to the square of the electron numbers and quadratically growing with the undulator length.

For the general case the optical field evolution equation [Eq. (7)] can be solved by Laplace transform:

$$
\mu \tilde{a}_{s}(\mu)=\frac{I_{T} \tilde{b}_{0}}{\mu+i \phi_{0}^{\prime}}+i\left(2 k_{u} \rho\right)^{3} \frac{\tilde{a}_{s}(\mu)}{\left(\mu+i \phi_{0}^{\prime}\right)^{2}} .
$$

The solution is

$$
\begin{aligned}
\tilde{a}_{s}(z) & =\sum \operatorname{Re} s \frac{I_{T} \tilde{b}_{0}\left(\mu+i \phi_{0}^{\prime}\right) e^{\mu z}}{\mu\left(\mu+i \phi_{0}^{\prime}\right)^{2}-i\left(2 k_{u} \rho\right)^{3}} \\
& =i\left(2 k_{u} \rho\right)^{3} \sum_{\substack{m=1 \\
m \neq l, k}}^{3} \frac{I_{T} \tilde{b}_{0} e^{\mu_{m} z}}{\mu_{m}\left(\mu_{m}+i \phi_{0}^{\prime}\right)\left(\mu_{m}-\mu_{l}\right)\left(\mu_{m}-\mu_{k}\right)} \\
& =\sum_{m=1}^{3} \frac{I_{T} \tilde{b}_{0} e^{\mu_{m} z}}{3 \mu_{m}+i \phi_{0}^{\prime}},
\end{aligned}
$$

where $\mu_{m}$ are roots of the well-known FEL cubic equation: $\mu\left(\mu+i \phi_{0}^{\prime}\right)^{2}=i\left(2 k_{u} \rho\right)^{3}$. When $\phi_{0}^{\prime} /\left(2 k_{u} \rho\right)<3 / \sqrt[3]{4}=$ 1.89 , the cubic equation has one imaginary root 
corresponding to the oscillatory mode of the optical field, and two complex roots with the real parts of equal magnitude but opposite sign, corresponding to the exponential amplifying mode and the exponential decaying mode, respectively [16]. [If $\phi_{0}^{\prime} /\left(2 k_{u} \rho\right)>1.89$ the cubic equation will have three imaginary roots']

At the resonant energy, the detuning parameter $\phi_{0}^{\prime}=0$, the roots of the cubic equation are $\mu=\left(2 k_{u} \rho\right) i^{1 / 3}$. Then the radiation power of the prebunched electron beam is obtained as

$$
\begin{aligned}
P= & \frac{2 b_{0}^{2} \rho P_{e}}{9}\left[\operatorname{ch}(\bar{z})-\cos \left(\frac{\sqrt{3}}{2} \bar{z}\right) \operatorname{ch}\left(\frac{\bar{z}}{2}\right)\right. \\
& \left.+\sqrt{3} \sin \left(\frac{\sqrt{3}}{2} \bar{z}\right) \operatorname{sh}\left(\frac{\bar{z}}{2}\right)\right],
\end{aligned}
$$

where $\bar{z}=z / L_{g}, L_{g}=1 / 2 k_{u} \sqrt{3} \rho$ is the power gain length. For comparison the optical power from the amplification of a seeding is (resonance $\phi_{0}^{\prime}=0$ ) [17]

$P=\frac{P_{0}}{9}\left\{1+4 \operatorname{ch}\left(\frac{\bar{z}}{2}\right)\left[\operatorname{ch}\left(\frac{\bar{z}}{2}\right)+\cos \left(\frac{\sqrt{3}}{2} \bar{z}\right)\right]\right\} \approx \frac{1}{9} P_{0} e^{\bar{z}}$.

The final exponential gain approximation is valid for $z>4 L_{g}$.

For a sufficiently long undulator $\left(z>4 L_{g}\right.$, see next section), the leading role is the exponential growth term corresponding to the root with positive real part. At the resonant energy it is $\mu_{1}=k_{u} \rho(\sqrt{3}+i)$, then from Eq. (11) the exponential growth can be given as

$$
\tilde{a}_{s}(z) \approx \frac{I_{T} \tilde{b}_{0} e^{k_{u} \rho(i+\sqrt{3}) z}}{3 k_{u} \rho(i+\sqrt{3})}, \quad P \approx \frac{1}{9} b_{0}^{2} \rho P_{e} e^{\bar{z}} .
$$

We can see it is equivalent to the exponential amplification [Eq. (13)] of a seeding power of $P_{0}=b_{0}^{2} \rho P_{e}$.

Notice that we can write the optical field equation [Eq. (1)] as $d \tilde{a}_{s} / d z=I_{T} \tilde{b}$, hence from Eq. (14) the evolution of the bunching factor in the exponential gain region is

$$
\tilde{b} \approx \frac{1}{3} \tilde{b}_{0} e^{\mu_{1} z} \quad \text { or } \quad b^{2}=\frac{1}{9} b_{0}^{2} e^{\frac{z}{L_{g}}}\left(z>4 L_{g}\right) .
$$

Therefore the emission power also can be written as

$$
P \approx b^{2} \rho P_{e} .
$$

Generally it has $b<1$. Considering the ideal case that the bunching factor reaches the ultimate $b \sim 1$, then the exponential gain reaches its maximum: $P_{s}=\rho P_{e}$.

We consider some schemes with the initial bunched electron beam.

The harmonic generation FEL schemes, including the superradiance FEL such as coherent harmonic generation (CHG) [18] and the high gain FEL such as high gain harmonic generation (HGHG) [19-20], are a special kind of prebunching mode, in which a seeding laser and a configuration of two undulators separated by a dispersive section are adopted. In the first undulator, which is tuned at the seed wavelength and called the modulation section, the electron energy is modulated owing to the strong optical field of the external seeding laser. Then in the dispersive section, with the stronger dispersive magnetic field, the electron microbunching is formed quickly. Next, in the second undulator, which is tuned at the $n$th harmonic of the seeding laser and called the gain section, the radiation at the wavelength is enhanced. The initial bunching factor in the gain section undulator is

$b_{0}=J_{n}\left(n D \frac{\Delta \gamma_{m}}{\gamma}\right) \exp \left[-\frac{1}{2}\left(n D \frac{\sigma_{\gamma}}{\gamma}\right)^{2}\right]=J_{n} f_{\gamma}$,

where $D=2 \pi\left(N_{1}+2 N_{d}\right), N_{d}$ is the dispersive parameter of the dispersive section, $N_{1}$ is the number of the modulation undulator periods; $\Delta \gamma_{m}$ is the maximum energy modulation of the electron beam by the seeding laser, $\sigma_{\gamma}$ is the energy spread of the electron beam. We denote the Bessel function factor and the energy spread factor in Eq. (17) as $J_{n}$ and $f_{\gamma}$ respectively. The corresponding radiation power of the HG-FEL can be given by substituting Eq. (17) into Eq. (12).

When the length of the gain section undulator is shorter than four gain lengths, the radiation is the CHG [21]. Substituting Eq. (17) into Eq. (8), the radiation power of CHG FEL is

$$
P=\left(2 k_{u} \rho L\right)^{2} J_{n}^{2} f_{r}^{2} \rho P_{e} .
$$

When the length of the gain section undulator is longer than four gain lengths, the evolution of the optical field becomes the HGHG process [21]. Substituting Eq. (17) into Eq. (14), the optical power of HGHG is

$$
P \approx \frac{1}{9} J_{n}^{2} f_{r}^{2} \rho P_{e} e^{\frac{z}{L_{g}}}
$$

For the echo-enabled harmonic generation (EEHG) scheme [22] the initial bunching factor in the gain section can be written as

$b_{n}=J_{n+1}\left(n \frac{\Delta \gamma_{m 2}}{\gamma} D_{2}\right) J_{1}\left[\frac{\Delta \gamma_{m 1}}{\gamma}\left(n D_{2}-D_{1}\right)\right] e^{-\frac{\sigma_{\gamma}^{2}}{2 \gamma^{2}}\left[n D_{2}-D_{1}\right]^{2}}$.

Here $D_{1}=4 \pi\left[N_{d 1}+\left(N_{1}+N_{2}\right) / 2\right], D_{2}=4 \pi\left(N_{d 2}+N_{2} / 2\right)$, are the dispersive intensities of the first and the second stage of EEHG, respectively. Taking $D_{1} \Delta \gamma_{m 1} / \gamma \approx$ $n \pi$ and $n D_{2} \Delta \gamma_{2} / \gamma=n+1+0.81(n+1)^{1 / 3}$, then for $\Delta \gamma_{1} / \sigma_{\gamma} \gg 1$, it has $b_{n} \approx 0.39 /(n+1)^{1 / 3}$. Substituting it into Eq. (12), the optical power of EEHG can be given. 
In the exponential gain region $\left(z>4 L_{g}\right)$, from Eq. (14) the optical power is

$$
P \approx \frac{1}{60} \frac{1}{(n+1)^{2 / 3}} \rho P_{e} e^{\frac{z}{L_{g}}}
$$

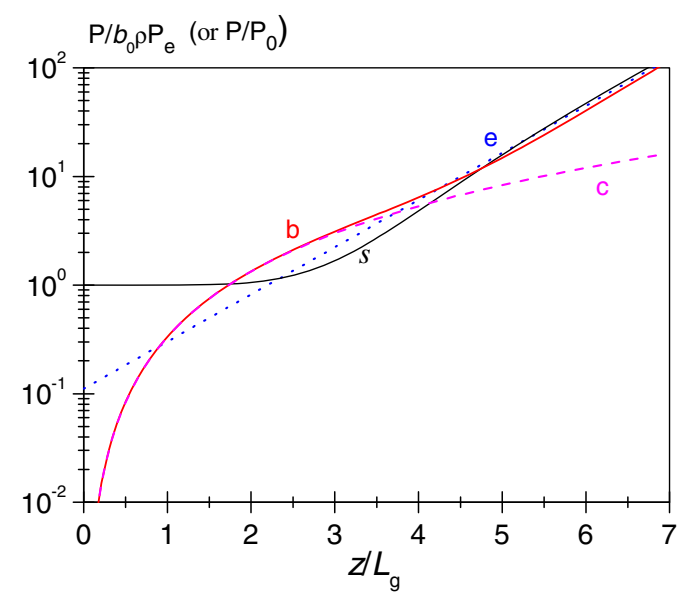

FIG. 1. The optical power growing vs undulator length $\left(\phi_{0}^{\prime}=0\right)$. The red line (b): the prebunched mode [Eq. (12)]. The pink dashed line (c): the coherent spontaneous emission [Eq. (8)]. The blue dot line (e): the exponential gain formula [Eq. (14)]. The black line(s): the seeded amplifier mode [Eq. (13)].
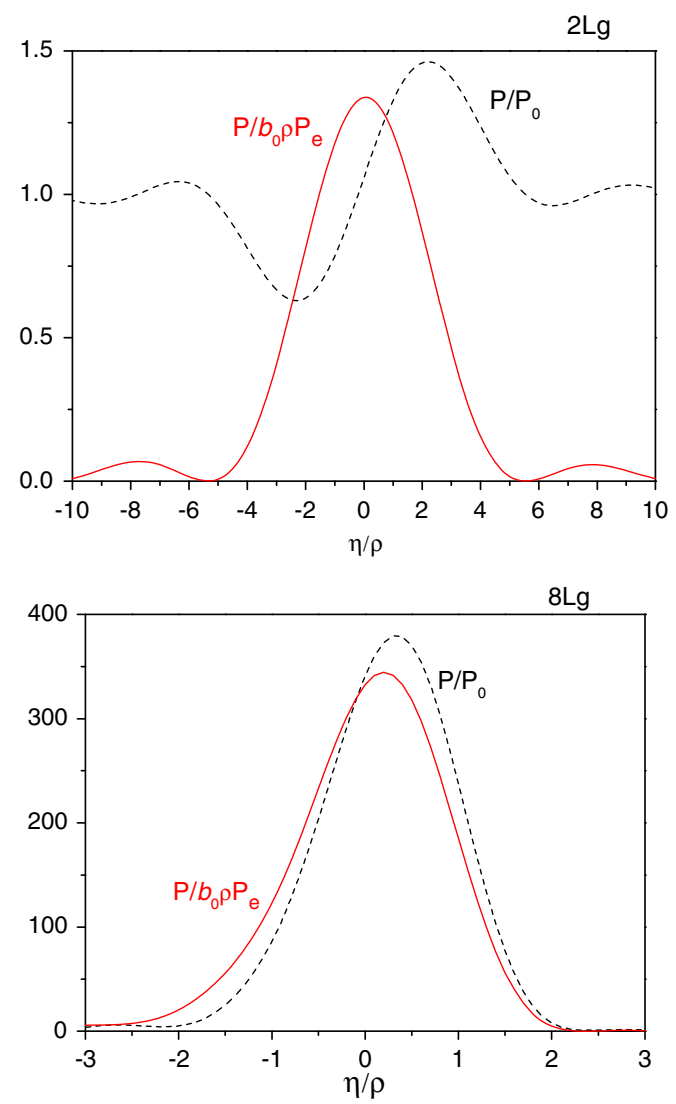

\section{DISCUSSION}

The variation of the optical power with the undulator length for the prebunched electron beam [Eq. (12)] is plotted in Fig. 1 (curve $b$ ). We can see that when $L<4 L_{g}$ it is just the coherent spontaneous emission [Eq. (8), curve $c$ ]; when $L>4 L_{g}$ it becomes the exponential growth of the stimulated emission [Eq. (14), curve $e$ ]. For comparison, we also plot the optical power of the seeded FEL amplifier [Eq. (13), curve $s$ ]. It is shown that the large difference is at the initial stage $\left(z<4 L_{g}\right)$. The optical power induced by the electron initial bunching is developed from zero, its exponential growth is the same as the seeding mode.

Using Eq. (11) we give the emission intensity variation with the detuning parameter $\left(\eta=\delta \gamma / \gamma=\phi_{0}^{\prime} / 2 k_{u}\right)$ of the prebunching mode for different gain lengths (Fig. 2). The case of the seeding mode [16] is also shown for comparison. It can be seen that the two cases have the larger difference in the initial stage and tend to identical when entering the exponential gain region $\left(z>4 L_{g}\right)$.

For the optical field growth induced by the prebunching electron beam, the detuning parameter corresponding to the maximum growth is initially at resonance $\eta_{m} / \rho \approx 0$ for the coherent spontaneous emission, then as the length of the undulator increases it becomes larger and remains at about $\eta_{m} / \rho \approx 0.1$ for the exponential gain. However, the growth
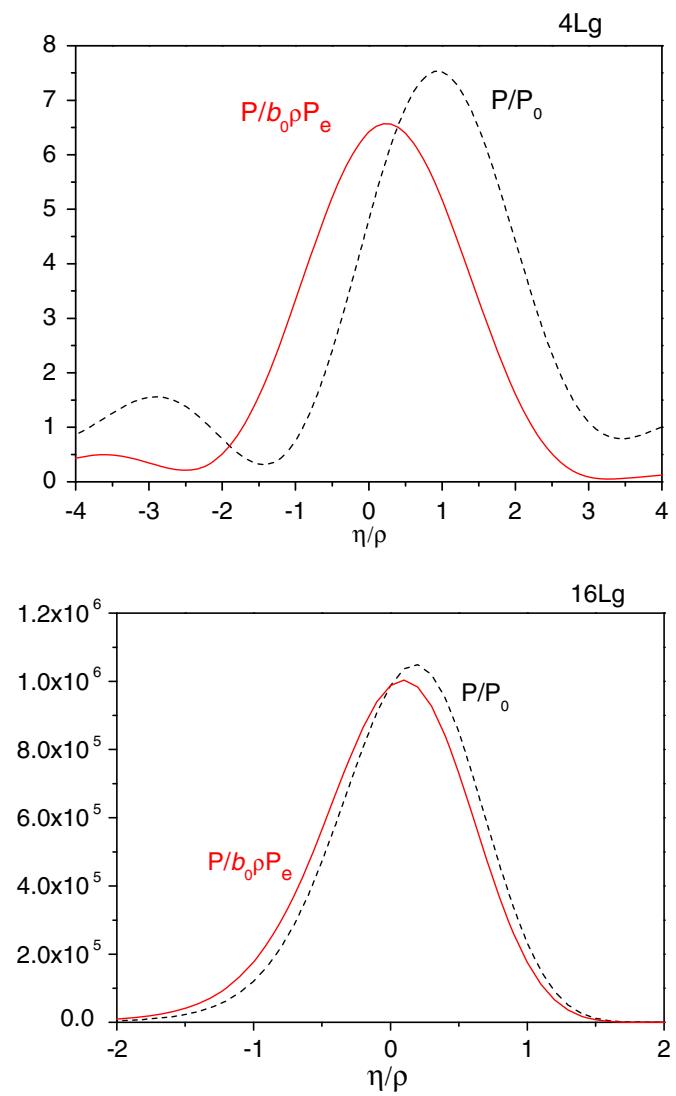

FIG. 2. The variation of the emission intensity with the detuning parameter for different interaction length. The solid line: prebunching mode; the dashed line: seeding mode. 


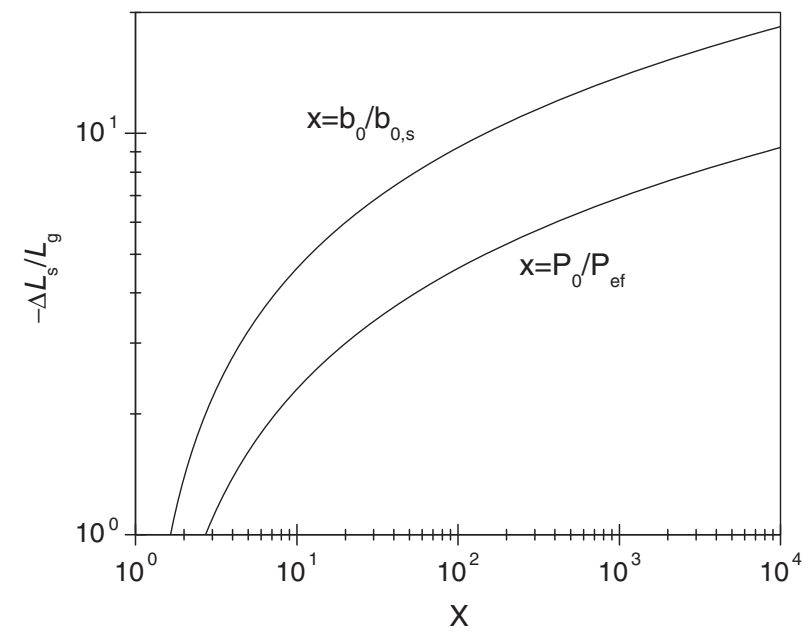

FIG. 3. Shortened saturation length with initial bunching [Eq. (22)] and seed power [Eq. (23)].

at resonance differed little from the maximum growth, therefore the expression of the optical field at resonance [Eq. (12)] can be used to describe the maximum growth approximately. The gain bandwidth of the prebunching mode decreases from $\Delta \eta / \rho \approx 10 L_{g} / L$ (FWHM) for the coherent spontaneous emission to $\Delta \eta / \rho \approx 1$ for the exponential gain near saturation.

For the optical field growing from the seeding, the initial stage is the small signal gain region, the detuning parameter corresponding to the maximum growth and the gain bandwidth are $\eta_{m} / \rho=2.6 / 4 \pi N$ and $\Delta \eta / \rho \approx 6.6 L_{g} / L$, respectively. Both of them decrease with increasing of the undulator length [16].

The saturation length of the exponential gain for the prebunched mode can be written as

$$
L_{s}=L_{s 0}-2 \ln \left(\frac{b_{0}}{b_{0, s}}\right) L_{g},
$$

where $L_{s 0}$ is the saturation length of self-amplified spontaneous emission (SASE), $b_{0, s}$ is the equivalent initial bunching factor of SASE. Typically $b_{0, s}$ is smaller than $10^{-3}$, therefore, even an initial bunching factor of not too large, e.g. $0.5 \%$, can be expected to overwhelm the shotnoise induced bunching of SASE, and substantially decrease the saturation length (Fig. 3) [8].

Similarly, the saturation length for the amplifier mode (the seeding laser is amplified directly) is

$$
L_{s}=L_{s 0}-\ln \left(\frac{P_{0}}{P_{e f}}\right) L_{g},
$$

where $P_{0}$ is the power of the seeding; $P_{e f}$ is the effective initial power of SASE, it is about the spontaneous radiation on two exponential gain lengths and has $P_{e f}=b_{0, s}^{2} \rho P_{e}$.

\section{SUMMARY}

From the FEL equations, we have analyzed the emissions of the prebunched electron beam, including the coherent spontaneous emission and the self-amplified stimulated emission. For the spontaneous emission, neglecting the phase changes due to the interaction of the electron with the radiation field we give the formula of coherent spontaneous emission, the power of which is proportional to the square of the initial bunching factor and of the undulator length. For the general emission case of the prebunched electron beam, we deduced the evolution equation of the radiation field. Then for the resonant case, we obtained the analytical expression of the emission power; the application scope of it is from the low gain regions to the high gain regions. We find that it is just the coherent spontaneous emission when the undulator length is shorter than four gain lengths, and conversely, it is the exponential gain of the self-amplified stimulated emission. For the nonresonant prebunched electron beam, we show the variations of the emission intensity with the detuning parameter for different interaction length. We also compared the radiation field characters of the prebunched electron beam with that of the seeded FEL amplifier, such as the evolution of the optical field, the saturation length, the variation of the detuning parameter corresponding to the maximum gain and the variation of the gain bandwidth with the interaction length.

The analytical results presented here will provide help to the design and analysis of a THz FEL or X-FEL devices with the prebunching mode, and also help to develop insights into understanding the FEL gain process. For further studies, the more practical influence factors should be considered, such as the space charge effect, the effect of the imperfect electron beam, the diffraction effect and so on.

\section{ACKNOWLEDGMENTS}

This work is partly supported by the National Nature Science Foundation of China under Grants No. 11675178, No. 11611140102, and No. 51627901.

[1] A. Gover, Superradiant and stimulated superradiant emission in prebunched electron-beam radiators part I: Formulation, Phys. Rev. ST Accel. Beams 8, 030701 (2005).

[2] G. Penn, M. Reinsch, and J. S. Wurtele, Analytic model of bunched beams for harmonic generation in the low-gain free electron laser regime, Phys. Rev. ST Accel. Beams 9, 060702 (2006).

[3] G. Dattoli, L. Giannessi, P. L. Ottaviani, and A. Segreto, FEL dynamics, electron-beam bunching and prebunching, Nucl. Instrum. Methods Phys. Res., Sect. A 393, 339 (1997). 
[4] I. Schnitzer and A. Gover, The prebunched free-electron laser in various operating gain regimes, Nucl. Instrum. Methods Phys. Res., Sect. A 237, 124 (1985).

[5] Y. Shibata, S. Sasaki, and K. Ishi, Coherent radiation from bunched electrons, and prebunched FEL in the millimeter wavelength region, Nucl. Instrum. Methods Phys. Res., Sect. A 483, 440 (2002).

[6] M. Yasuda, H. Hama, F. Hinode, K. Kasamsook, M. Kawai, A. Kurihara, K. Nanbu, Y. Shibasaki, and S. Takahashi, Development of $\mathrm{THz}$ light source using prebunched FEL, in Proceedings of the 30th International Free Electron Laser Conference, Gyeongju, 2008 (JaCoW, Gyeongju, 2008).

[7] Y.-C. Huang, Desktop megawatt superradiant free-electron laser at terahertz frequencies, Appl. Phys. Lett. 96, 231503 (2010).

[8] P. Freund, D. C. Nguyen, and B. Carlsten, Threedimensional analysis of prebunched electron beams in an X-ray free-electron laser, Phys. Rev. ST Accel. Beams 15, 030704 (2012).

[9] W.S. Graves et al., Compact XFEL light source, in Proceedings of the 35th International Free Electron Laser Conference, New York, 2013 (JaCoW, New York, 2013).

[10] Free-Electron Generators of Coherent Radiation, edited by S.F. Jacobs, H. S. Pilloff, M. Sargent III, M. O. Scully, and R. Spetzer, Physics of Quantum Electronics (Addison-Wesley, Reading, 1982), Vol. 5, pp. 7-9.

[11] C. A. Brau, Free Electron Lasers (Academic Press, New York, 1990).

[12] W. B. Colson, Classic Theory of FEL; J. B. Murphy, Introduction to FEL physics, in Laser Handbook, edited by W. B. Colson, C. Pellegrini, and A. Renieri (NorthHolland, Amsterdam, 1990), Vol. VI.

[13] A. He, L. Yang, and L. Yu, Introduction to high-gain FEL theory, in Synchrotron Light Sources and Free-Electron
Lasers, edited by E. J. Jaeschke, S. Khan, J. R. Schneider, and J. B. Hastings (Springer International Publishing, Switzerland, 2016), pp. 119-161, ISBN: 978-3-31904507-8.

[14] K.-J. Kim and Z. Huang, Introduction to the physics of free electron lasers, Lecture note, 2007; Review of x-ray freeelectron laser theory, Phys. Rev. ST Accel. Beams 10, 034801 (2007).

[15] W. B. Colson and A. M. Sessler, Free electron lasers, Annu. Rev. Nucl. Part. Sci. 35, 25 (1985).

[16] J. Qika, Analytical formula of free electron laser exponential gain for a nonresonant electron beam, Chin. Phys. C 39, 048101 (2015).

[17] G. Dattoli, M. Del Franco, M. Labat, P. L. Ottaviani, and S. Pagnutti, Introduction to the Physics of Free Electron Laser and Comparison with Conventional Laser Sources, Free Electron Lasers, edited by S. Varro, (InTech, Rijeka, Croatia, 2012), pp. 1-38, ISBN: 978-953-51-0279-3, available from http://www.intechopen.com/books/free-electronlasers/ free-electron-laser-devices-a-comparison-with-ordinarylaser-sources.

[18] J. M. Ortega et al., Ultraviolet coherent generation from an optical klystron, IEEE Quantum Electron. 21, 909 (1985).

[19] L. H. Yu, Generation of intense UV radiation by subharmonically seeded single-pass free-electron laser, Phys. Rev. A 44, 5178 (1991).

[20] L. H. Yu et al., First lasing of a high-gain harmonic generation free-electron laser experiment, Nucl. Instrum. Methods Phys. Res., Sect. A 445, 301 (2000).

[21] Jia Qika, Optical klystron and harmonic generation free electron laser, Phys. Rev. ST Accel. Beams 8, 060701 (2005).

[22] G. Stupakov, Using Beam Echo Effect for Generation of Short-Wavelength Radiation, Phys. Rev. Lett. 102, 074801 (2009). 